\title{
Visual Aided GPS Navigation for Autonomous Mobile Robots
}

\author{
MPS Bhatia \\ Professor, \\ COE Dep't, NSIT
}

\author{
Saurav Kumar \\ Faculty \\ COE Dep't, DTU
}

\author{
Akshi Kumar \\ Lecturer \\ COE Dep't, DTU
}

\author{
Amit Kothari \\ Student \\ COE Dep't, DTU
}

\begin{abstract}
Navigation based on GPS data has been the most commonly used methodology for the autonomous run of a mobile robot in indoor and outdoor environments. However, the reading of the GPS receivers fluctuates over a considerable range esp. in countries like India where there is a dearth of GPS signals and locally available correction table. So the commonly received value by GPS receiver can be accurate over a range of $10-15 \mathrm{mts}$ which is inadequate if the test results require accuracy. This paper introduces a technique for the development of a visual aided GPS navigation system for a mobile robot in which we have predefined visual landmarks for the various important landmarks which robot has to visit. In our approach, we have mounted a stereovision camera on the robot platform for image acquisition, real time object recognition, detection and local features extraction from images using Scale-Invariant Feature Transform (SIFT).

Robust software based on the Open Computer Vision Library (OpenCV) has been developed for handling images from a stereovision camera and determine local heading direction. Following the description of the navigation technique, real world experiments demonstrate the functionality of the presented approach thereby substantiating the validity of the algorithm.
\end{abstract}

The full text of the article is not available in the cache. Kindly refer the IJCA digital library at www.ijcaonline.org for the complete article. In case, you face problems while downloading the full-text, please send a mail to editor at editor@ijcaonline.org 